\title{
Successful removal of stented leads by using percutaneous approach
}

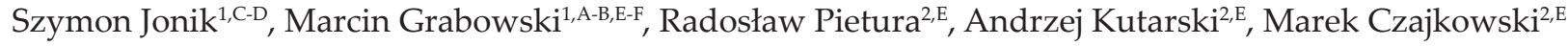 \\ A - Research concept and design, B - Collection and/or assembly of data, C - Data analysis and interpretation, \\ D - Writing the article, E - Critical revision of the article, F - Final approval of article \\ 1. 1st Department of Cardiology, Medical University of Warsaw \\ 2. Department of Cardiology, Medical University of Lublin
}

Address for correspondence:

Szymon Jonik, 1st Department of Cardiology, Medical University of Warsaw email: szymonjonik.wum@gmail.com

Marcin Grabowski, 1st Department of Cardiology, Medical University of Warsaw email: marcin.grabowski@wum.edu.pl Radosław Pietura, Department of Cardiology, Medical University of Lublin
email: radoslaw.pietura@umlub.pl

Andrzej Kutarski, Department of Cardiology, Medical University of Lublin email: kutarski.kardiolog@wp.pl

Marek Czajkowski, Department of Cardiology, Medical University of Lublin email: info@kardiochirurgia.lublin.pl

Received: 10.10 .2017

Revised: 08.12.2017

Accepted: 12.12.2017

Final review: 24.10 .2017

DOI: $10.24255 / \mathrm{hbj} / 81161$

\section{Key words:}

vascular surgery, venous occlusion, transvenous lead extraction, Stented leads, Superior vena cava syndrome

\section{Abstract}

Background: Nowadays we are implanting more cardiac implantable electronic device (CIED) systems with intracardiac leads. Unfortunately, sometimes when the lead remains in the vessel too long it adheres to its wall, causing venous occlusion, and the removal is problematic. Venous occlusion is treated with venoplasty with the stent remaining. The standards clearly recommend removing all leads before venoplasty and stenting. Stenting of leads into the vessel wall makes them practically indelible. In the literature, we found only one report of successful removal of veno-thoracic stented leads.

\section{Introduction}

The increased frequency of implantation of cardiovascular implantable electronic devices has also led to a rise in the number of lead extraction procedures. ${ }^{[1]}$

The indications for percutaneous lead extraction are infection, chronic pain at the implantation site, thrombosis and
Methods: We present two cases of patients who did not have all leads removed before venoplasty with stent implantation.

Results: All 4 stented leads in the two patients were successfully extracted using a percutaneous approach. The oldest pacing lead extracted was 17 years old, while the oldest stent was 11 years old.

Conclusion: Removal of stented leads is a serious challenge, but may be safe and effective if minimally invasive equipment is used.

venous stenosis/occlusion, nonfunctional leads and functional leads that represent a risk to the patient if left in place.

Lead-related venous occlusion is observed in $15-40 \%$ of patients and is an inherent complication of endocardial pacing. The problem is not only because of the difficulty or inability to implant additional leads, but also because of hemodynamic 
consequences such as superior vena cava syndrome (SVCS). SVCS occurs in the absence of adequate collaterals, and the resulting elevated venous pressure in the upper body leads to edema of the head, neck, and upper extremities, or upper limb edema (if axillary vein occlusion is present). Treatment of large venous stenosis is cumbersome and involves balloon angioplasty of the vein, usually with simultaneous stent placement. It is difficult to achieve a decent result of this procedure, because it is necessary to carry a lead for the venoplasty and stenting, and to do so, it is necessary to obtain venous access by removing the electrode.

Extraction of the lead is not easy either, and the difficulty is directly proportional to the severity of the fibrotic and adhesive scar tissue that encases the lead, vascular endothelium, and cardiac chambers. In long-standing leads, calcification can occur. ${ }^{[2]}$

Although venoplasty and stenting large veins became widespread in the 1990s, only a few centers, since quite recently, have had adequate experience with both techniques. So, there existed a situation in which the rebuilding of venous flow with intravenous plasty of the superior vena cava (SVC) was performed in patients with left leads. The perioperative effect of this procedure was good, but long-term follow-up has shown this to be wrong, and today no leads are stented to the SVC wall. However, to date there have been patients who did not have all the leads extracted. Here, we present two cases of such patients.

\section{Case reports}

Patient No. 1, male, was admitted to the Cardiology Unit because of dysfunction of pacing leads. A dual chamber pacemaker with unipolar leads was implanted by the left cephalic vein many years earlier because of third-degree atrioventricular block. Then, due to dysfunction of the old leads, 2 bipolar leads were implanted by the left subclavian vein. Later, because of dysfunction of one of the leads on the left side, a new VDD pacemaker was implanted on the right side (the leads on the left side were abandoned). Overall, the patient had 5 electrodes in the SVC. Since 2000, increasing symptoms of SVCS - facial and neck edema and headaches - have been reported by the patient. The venography in 2001 revealed complete occlusion of the anonymous vein and significant double stenosis of the SVC (Figure 1. A, B).

We successfully removed 4 unnecessary leads on the left side. There were no complications. Unfortunately, the venous flow was not restored. We decided on venoplasty of the SVC with stent implantation (Figure 1.C). The procedure was successful (Figure 1.D), but the patient stopped acenocoumarol and the symptoms of SVCS reappeared in 2007 (Figure 2.A, B). Based on the available X-ray image, we assumed that the lead was inside the stent. So, we decided to remove the lead to allow a stent reflow procedure to be performed. A new DDD pacemaker was implanted through the femoral approach. The pacemaker was implanted in the abdominal wall. In 2008 we finally extracted the VDD lead (we also

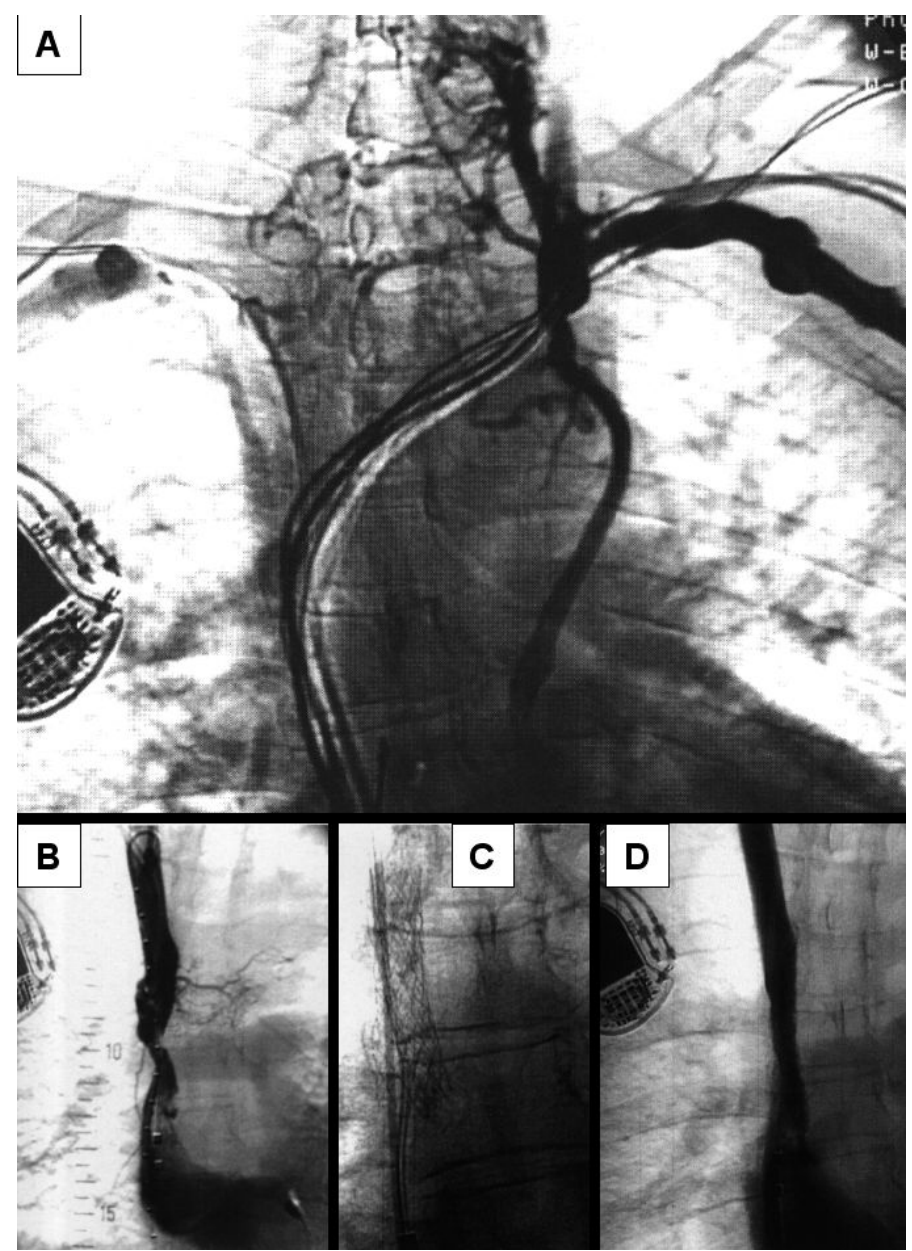

Figure 1.

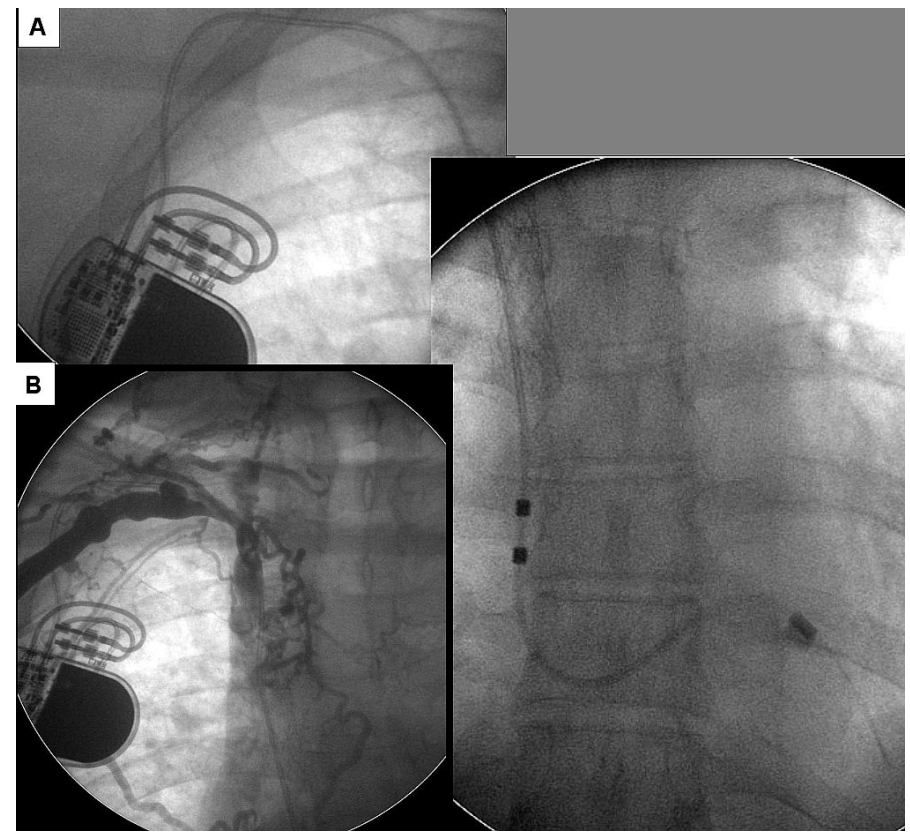

Figure 2.

successfully introduced the lead and catheter) (Figure 3.A, B). For extraction of the lead we used a standard stylet with green Byrd's dilators (size B; Inner Sheath ID/OD 10.0/12.1 Fr; Outer Sheath ID/OD 13.1/15.2; Length Inner/Outer 38/33 cm). 


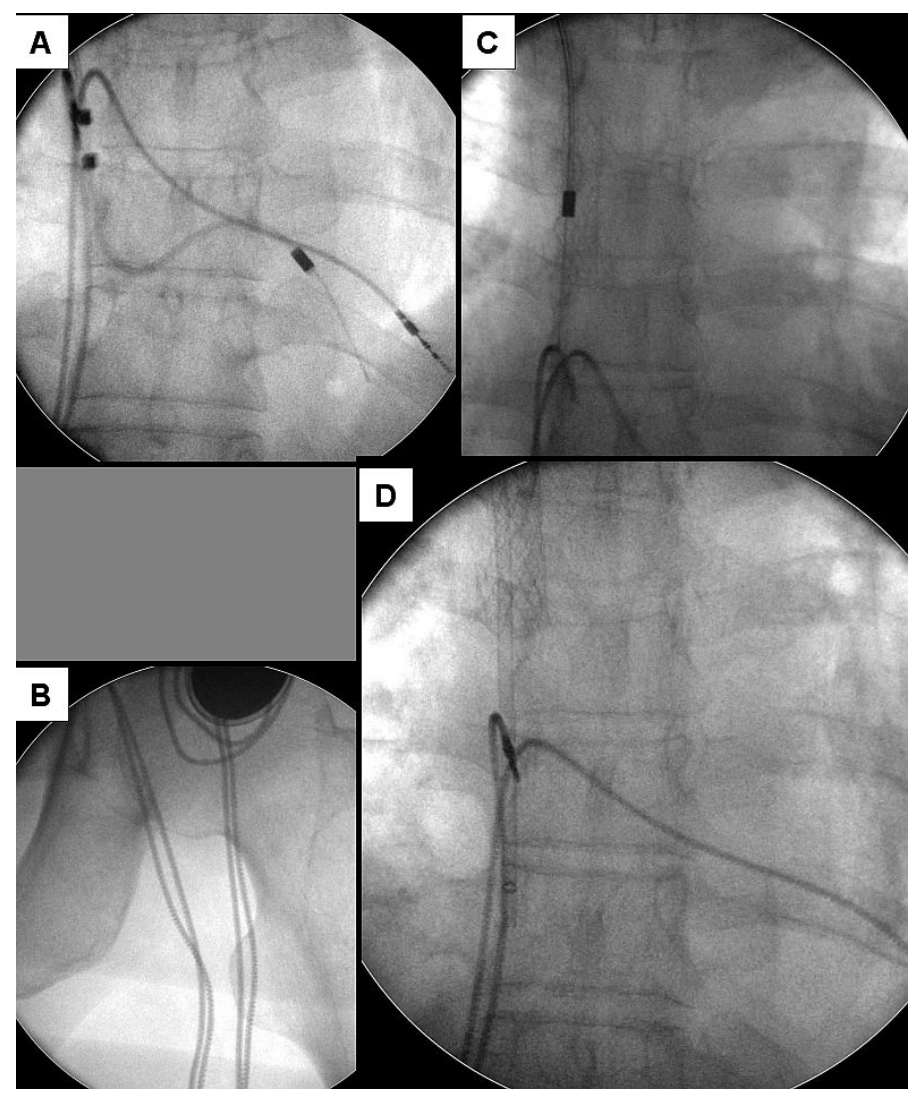

Figure 3.

To leave the channel for a while we introduced via the Byrd dilator a longer catheter, and after it an old Attain sheath, later removed. The stent remained intact. After the lead removal the patient was ready for the stent-releasing procedure. During the procedure, it turned out that from the beginning the lead was out of the stent and we were able to remove it even though it was adhered to by a very old stent. The patient did not consent to further treatment and refused to undergo venous bypass surgery (right brachiocephalic trunk to right atrium). The patient is still alive today in good health.

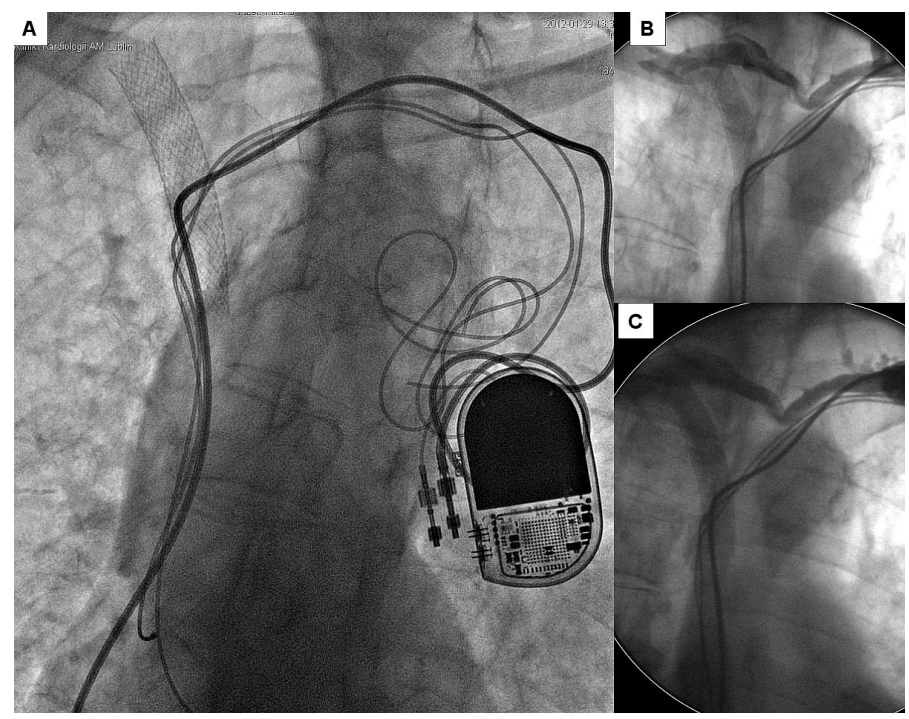

Figure 4.
Patient No. 2, female, had in 1982 due to sick sinus syndrome (SSS) a VVI pacemaker on the right side of the chest implanted. The patient underwent replacement due to an elective replacement indication in 1993. In addition, the patient has been using the AAI system since 1986. Unfortunately, we do not have access to the patient's medical records and we do not know where and for what reason the AAI was implanted. Because of the pacemaker syndrome in 1995, the patient was implanted with a DDD system on the left (TIR 60 UP and DY 60 UP) - there was already an occlusion on the right (Figure 4.A, B, C). In 2001, the patient reported typical symptoms for SVCS. We therefore extracted surgically an unnecessary ventricular lead on the right side and performed surgery of the right brachiocephalic trunk with stent implantation (Figure 4.A).

We left a tiny fragment of the lead in the pocket on the right side, but because of the high risk of infection we were mainly concerned with limiting the time of surgery to the minimum. In 2005 there was a failure of the RV lead on the left side, so we urgently implanted a new thick (screwdriver) right ventricular lead, while the old ventricular lead was abandoned.

In 2011 the DDD system on the left side was replaced. Our patient presented symptoms of infective endocarditis recurrences of fever, elevated OB. In venography, the stent was still patent and provided proper drainage of blood from

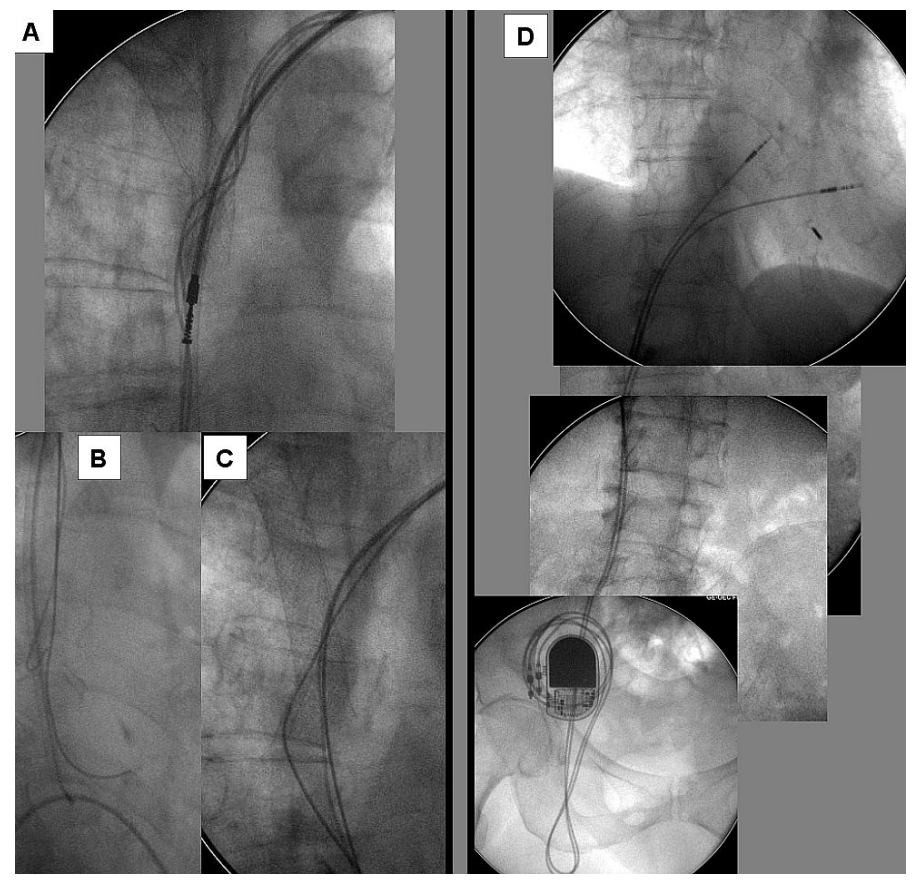

Figure 5 .

the upper limbs and head. We decided to remove all the leads with only 3 weeks left RAA and RVA on two screwdrivers. Despite the stented old device leads in the SVC, the removal of all the leads was successful (Figure 5.A, B, C). In this case for extraction of the leads we also used a standard stylet with green Byrd's dilators (size B; Inner Sheath ID/OD 10.0/12.1 Fr; Outer Sheath ID/OD 13.1/15.2; Length Inner/Outer 38/33 cm). After 3 weeks the patient had a new DDD system implanted via the right femoral vein (Figure 5.D). The device worked 
properly during the check-up half a year after the procedure. Unfortunately, the patient died from oncological causes one year after the procedure.

In all images after removing the electrodes the catheter is visible. Leaving the catheter is a standard additional emergency central access - in the case of tamponade, rapid administration of fluids and blood is possible. The catheter was removed a moment later after the procedure.

\section{Results}

All leads (4) were successfully extracted by the percutaneous approach. In the case of patient No. 1, the VDD electrode at the time of removal was 12 years old, while the stent was 7 years old. In the case of patient No. 2 we removed (2012) 2 stented leads from 1995 (17 years old), while the stent was 11 years old (the third electrode from 2005 was implanted outside the stent).

\section{Discussion}

Treatment of large vein occlusion involves venoplasty or stenting. The vascular surgery of veins requires a highly skilled surgeon and has a good effect only for short term; the veins close again after some time. The surgery is not technically easy and may involve many complications. Endovascular stenting is more effective: for SVC syndrome it has a high technical success rate, greater than $95 \%$ in some series. ${ }^{[3]}$ SVC stenting can usually be performed under local anesthesia, and the most crucial stage in the procedure is to cross the stenotic lesion. In cases of near total occlusion, bi-directional cannulation (femoral vein and jugular vein approach) can be helpful. In cases of bilateral brachiocephalic vein thrombosis, restoring flow in one vein is usually sufficient to relieve symptoms on both sides. ${ }^{[4,5]}$ This is likely explained by the fact that the collaterals would cross the midline and drain the contralateral vein. Cannulation of both veins is not only time-consuming but is also technically difficult and not cost effective. ${ }^{[4]}$

Stenting may also result in less serious complications: major complications for SVC stenting have been reported, but only rarely. ${ }^{[3]}$ Complications have included mainly SVC rupture, cardiac tamponade, pulmonary embolism and hemorrhage. Other minor complications such as puncture site hematoma, chest pain and hemoptysis have also been reported. Stent migration into the right atrium or even right ventricle may appear as another, extremely rare problem. The most common late complication following SVC stenting is in-stent restenosis or thrombosis, which has been found in $0-40 \%$ of patients. ${ }^{[3,4,5]}$

Stenting gives a favorable long-term effect if anticoagulation is effective. While there is no consensus in the literature regarding the effectiveness of anticoagulation therapy in preventing SVC stent thrombosis, anticoagulants or anti-platelet agents are generally prescribed for a period of 1-9 months postoperatively ${ }^{[3,4,5,6]}$ Currently, no large clinical trials comparing antiplatelet agents vs. anticoagulants after SVC stenting are underway.
It is necessary, however, to avoid stenting of leads to the walls of large vessels, removing all of them before the recanalization attempt. When the leads are already in place, permanent, effective anticoagulation is required and additionally lead function monitoring because of the potential risk of damage of the leads by the sharp edge of the stent, which is particularly dangerous for pacemaker-dependent patients.

Moreover, stented leads are known to be very difficult to extract. The removal is hazardous and can result in rupture of the vein behind the stent. Perforation of the SVC or brachiocephalic vein is a potentially fatal complication of such lead extraction. Depending on its location, perforation can result in pericardial, mediastinal, or pleural bleeding. Tears below the pericardial reflection leading to pericardial bleeding and tamponade are the most common causes of hemodynamic collapse associated with lead extraction if conventional tools are used. Utility of laser sheaths is largely restricted to upper location of the vein wall damage $\mathrm{e}^{[]]}$. In a single-center study, Brunner et al. reported a mortality rate of $31.2 \%$ for SVC lacerations during lead extractions at the Cleveland Clinic, a high-volume extraction center with significant resources and comprehensive rescue protocols in place. ${ }^{[8]}$

In the case of venous stenting, for its function and vascular condition the best solution is no leads in the vessel, and if they are present already, the best option is to introduce them by using a femoral or epicardial approach. ${ }^{[9]}$

Although more data and reporting of outcomes are required for confirmation, conclusions from the initial observations seem generally optimistic.

\section{Conclusion}

Treatment of leads related to venous occlusion concerning SVC and brachiocephalic veins still remains a clinical challenge. The team have to possess considerable experience in the transvenous extraction of leads before stenting and - for management of mistakenly stented leads - extraction of such leads as well. Lead extraction teams should prepare to manage complications by planning for the possibility of hemodynamic collapse and ensuring familiarity with rescue protocols.

The removal of a stented rooted lead is technically feasible and safe if the most secure device, which is the Byrd telescopic catheter system, is used and the intervention is performed by an experienced operator.

\section{References}

1. Hauser RG, Katsiyiannis WT, Gornick CC, Almquist AK, Kallinen LM. Deaths and cardiovascular injuries due to device-assisted implantable cardioverter-defibrillator and pacemaker lead extraction. Europace 2010;12:395-401.

2. Jon A. Grammes, Christopher M. Schulze, Mohammad Al-Bataineh, et al. Percutaneous pacemaker and implantable cardioverter-defibrillator lead extraction in 100 patients with intracardiac vegetations defined by transesophageal echocardiogram. Journal of the American College of Cardiology, Volume 55, Issue 9, 
3. March 2010, 886-894.

4. de Gregorio Ariza M, Gamboa P, Gimeno MJ, Alfonso E, Mainar A, Medrano J, et al. Percutaneous treatment of superior vena cava syndrome using metallic stents. Eur Radiol 2003;13:853-862.

5. Ganeshan A, Hon LQ, Warakaulle DR, Morgan R, Uberoi R. Superior vena caval stenting for SVC obstruction: current status. Eur J Radiol 2009;71:343-349.

6. Jackson JE, Brooks DM. Stenting of superior vena caval obstruction. Thorax 1995;50:S31-36.

7. Warner $P$, Uberoi R. Superior vena cava stenting in the 21st century. Postgrad Med J 2013;89:224-230.

8. Henrikson CA, Brinker JA. How to prevent, recognize, and manage complications of lead extraction. Part III: procedural factors. Heart Rhythm 2008;5:1352-1354.

9. Brunner MP, Cronin EM, Wazni O, et al. Outcomes of patients requiring emergent 210 surgical or endovascular intervention for catastrophic complications during transvenous 211 lead extraction. Heart Rhythm. 2014;11(3):419-425.

10. Andrzej Kutarski, Maciej Polewczyk, Radosław Pietura. A rare complication of transvenous lead extraction - pulmonary embolization with a broken distal lead fragment. Heart Beat 2016;1:31-34. 